\section{OPEN ACCESS \\ Journal of \\ Intelligence}

ISSN 2079-3200

www.mdpi.com/journal/jintelligence

Editorial

\title{
Open Access Intelligence
}

\author{
Paul De Boeck \\ Department of Psychology, The Ohio State University, Columbus, OH 43210, USA; \\ E-Mail: deboeck.2@osu.edu
}

Received: 20 November 2012 / Accepted: 12 December 2012 / Published: 17 December 2012

The Journal of Intelligence is a journal devoted to the study of human intelligence. Intelligence is a remarkable and highly intriguing phenomenon, and a core feature of our being humans. Understanding our intelligence is a major part of understanding ourselves. Human intelligence is studied from many perspectives and for different purposes. The basic issues in this study can be organized as follows: where does intelligence come from, what is it, what are its correlates and consequences? Where does it come from leads to more specific questions such as: how has it evolved through natural selection, what are the genetic and environmental factors influencing its development, how is intelligence wired in the brain and which brain processes does it involve? What is it can be understood in a cognitive process and in an ability sense and leads to further questions such as: is it differentiated from knowledge, where is it located in cognitive architecture systems, how shall it be measured, what is the structure of its individual differences, what are the different types, how to think of emotional, social, and practical intelligence, can artificial intelligence and possibly other types of intelligence help to understand human intelligence, how does artificial intelligence differ from human intelligence? What are its correlates and consequences implies questions such as: how does it relate to academic success and careers, to health and longevity, to relationships and trust, to attitudes and opinions?

On the one hand, the Journal of Intelligence focuses on human intelligence. It is the most intriguing form of intelligence, also from an artificial intelligence point of view. On the other hand, the scope of the journal is broader than human intelligence in the sense that also the study of other types of intelligence can help us understand human intelligence. The aim, scope, and focus of the journal are further explained towards the end of this editorial.

Perhaps the most prominent paradigm in the study of human intelligence is the psychometric paradigm. It has led to the IQ and $g$ or general intelligence. The IQ is the most popular measure in psychology, in research and in practice, and it is well-known by the general public. The concept of general intelligence, its factor-analytic equivalent $g$ and its associated IQ measure, are empirically 
highly powerful because of the many and important correlates. In an editorial in the journal Intelligence, Sternberg and Kaufman describe three important intelligence research topics, all three in terms of $g$ : “(a) finding new correlates of $g$ and (b) understanding better the nature of $g$, (c) expanding our conceptions of intelligence beyond $g$ " [1]. The authors suggest that intelligence research should tilt towards (b) and (c), since there is ample evidence now for what the correlates are (a). Yet what the underlying mechanisms are for the correlations is still an unanswered question.

The quest for the nature of $g$ is a specification of the more general question what the nature of intelligence is, the Holy Grail of the discipline. Various lines of research have been followed and are still being followed in this quest. For example, a quite different paradigm in the study of human intelligence than the psychometric approach is the information processing and cognitive architecture approach. While the psychometric approach is based on individual differences and correlations between tests, the cognitive processes and architecture approach is based on a theoretical and empirical analysis of perceptual and cognitive activities, memory and language, and tasks related to these. Cognitive process and architecture approaches have led to notions such as encoding and retrieval, short-term storage, working memory, central executive, etc., and corresponding brain structures and functions. The gap between the psychometric approach and the cognitive approach is bridged by studies in which individual-difference measures of the cognitive notions are correlated with the IQ, recently also in combination with structural and functional brain imaging data, and other brain research techniques.

An alternative route in search for the nature of intelligence is the route of elementary cognitive tasks and mental chronometry, with choice reaction times as the prototypical more specific paradigm. Various mental chronometry measures have been investigated and their neural basis has been explored. Important questions are where this has led and how the findings relate to the results of higher-order cognition studies from the cognitive processes and architecture approach.

Finally, there has been and there still is ongoing research motivated by the hope to pinpoint the genes of intelligence and the location of intelligence in the brain. Based on the findings thus far, there is not much hope this research will yield simple and specific answers. This relative lack of success makes the question all the more interesting. It seems that the answers will be as complex as intelligence itself: a multiple and interactive genetic basis and a distributed location in complicated and layered networks of the brain. A thrilling question is how this high complexity can be reconciled with the unitary $g$ and the rather simple IQ measure? A possible answer is that intelligence is an emergent property of the integration of the output of multiple brain regions.

Apart from the enduring search for the nature of intelligence, some specific phenomena have attracted the attention of researchers, because of their societal impact and relevance for how to think of intelligence. Perhaps the most intriguing among these is the Flynn effect, the rise of IQ through generations. Is it a true effect or a spurious finding? And what is the explanation? Did the rise of IQ scores come to an end or does it continue?

Because extreme forms of a phenomenon can help us understand its nature, and because rather extreme forms of intelligence can have serious consequences for the persons in question, it is of interest to investigate intellectual disabilities and intellectual giftedness. Both can make life more difficult and both may require forms of special education, although for different reasons. The present day society is complex, a huge challenge for all of us and especially for persons with intellectual 
disabilities. On the other hand, high potential persons are needed for the innovations that are so crucial for science, technology and medicine, for the economy, and to find solutions for the grand challenges for our societies.

Also the development and possible decline of intelligence is a highly intriguing issue. How does intelligence develop through life, is it a process of diversification or of integration? How much can it be stimulated, how does its development react to unfavorable situations? What do the growth curves of different intelligences look like and which aspects of intelligence start to decline with aging, and why?

Although the vast bulk of human intelligence studies are focused on cognitive intelligence, also other forms of intelligence have received attention in the literature and have stimulated exciting research, such as on emotional intelligence, social intelligence, and creativity. The work is not finished. Can these intelligences be investigated with the common paradigms for cognitive intelligence? Can they be measured properly? How important are they and for which criteria? There are of course answers to these questions, but not everyone is convinced that they are good answers.

Human intelligence was smart enough to invent artificial intelligence, as a support or replacement, for practical or efficiency reasons. Highly interesting research is going on in the overlap between human and artificial intelligence, which will most likely yield further insights in human intelligence. The year 2012 is the year of the Alan Turing centennial, the father of artificial intelligence. Can Turing machines compete with human intelligence, and in which respects they can and cannot? Does human intelligence have distinctive features? How much and what can artificial intelligence learn from how the brain functions? A booming field is brain informatics, a high multidisciplinary discipline where brain scientists, informatics scientists, and cognitive scientists meet and collaborate.

Because the study of human intelligence is often highly quantitative and based on formal models, it is important to keep high standards for the quantitative and modeling aspects of theorizing on intelligence and for the analysis of data. Examples are psychometric models such as item response models and advanced structural equation modeling, methods to deal with multilevel and spatial dependency in the cross-national or cross-regional studies, mathematical models for cognition, and goodness of fit approaches for these models.

The aim of the open access Journal of Intelligence is to offer a forum for the report of a large variety of studies, larger than could be sketched here, for the discussion of new ideas and for debates on important and sometimes controversial issues. The journal will publish original empirical and theoretical articles, state-of-the-art articles and critical reviews, case studies, original short notes, commentaries, and letters. Our aim is to publish an open access journal that moves the study of human intelligence forward in its many aspects. The topic of the contributions is expected to be relevant for the study of human intelligence. All topics covered in this editorial are welcomed and also other topics as far as they shed light on aspects of human intelligence. The journal does not want to promote or even suggest a definition of intelligence but wants to be open to all kinds of views and approaches instead.

We encourage authors to document their results in as much detail as possible, not in the actual paper, but as supplementary material. The full experimental and data analysis details must be provided so that the results can be reproduced and so that other researchers can better build on earlier work. 
Detailed descriptions of the procedure, software code and output of analyses must be made available in order to be deposited, unless in contradiction with privacy or security regulations or intellectual property rights. Authors will be encouraged to affirm in print "We report all data exclusions (if any), all manipulations, and all measures in the study, as well as how we determined our sample size." This openness has multiple advantages: transparency, facilitation of secondary analysis by other researchers, opportunities for collaboration, prevention and correction of possible mistakes [2].

We plan special issues on key issues and hot topics. The editorial board has broad and high-level expertise in the domain of intelligence and will ensure the quality and frontier character of the contributions. The success of the journal depends also on your participation in this endeavor, through the submission of papers, through your contribution as a reviewer, through suggestions you may have for special issues and topics to be covered, and through your feedback on our publication policy.

\section{References}

1. Sternberg, R.J.; Kaufman, S.B. Trends in intelligence research. Intelligence 2012, 40, 235-236.

2. Wicherts, J.M.; Bakker, M. Publish (your data) or (let the data) perish. Why not publish your data too? Intelligence 2012, 40, 73-76.

(C) 2013 by the authors; licensee MDPI, Basel, Switzerland. This article is an open access article distributed under the terms and conditions of the Creative Commons Attribution license (http://creativecommons.org/licenses/by/3.0/). 